九州大学学術情報リポジトリ

Kyushu University Institutional Repository

\title{
A CLOSED SEQUENTIAL PROCEDURE SELECTING THE BEST POPULATION IN A FAMILY OF POPULATIONS WITH ONE PARAMETER EXPONENTIAL DISTRIBUTIONS
}

Nomachi, Yukio

Miyazaki University

https://doi.org/10.5109/13023

出版情報: 統計数理研究. 12 (3/4), pp. 21-34，1967-03. Research Association of Statistical Sciences

バージョン：

権利関係 : 


\title{
A CLOSED SEQUENTIAL PROCEDURE SELECTING THE BEST POPULATION IN A FAMILY OF POPULATIONS WITH ONE PARAMETER EXPONENTIAL DISTRIBUTIONS
}

\author{
By \\ Yukio NoMACHI \\ (Miyazaki University)
}

\section{$\S 1$. Summary.}

The problem to select the best population in some specified sense from several assigned populations in the light of samples drawn from them is very important in practical situations. An experimenter who is faced with this problem may select the best population according to a certain statistical procedure in view of the informations supplied by samples drawn from these populations.

In this connection several different statistical procedures have been introduced by many authors such as Bahadur [2], Bahadur and Robbins [3], Bechhofer [4], [5], Bechhofer and Blumenthal [6], Bechhofer, Dunnett and Sobel [7], Dunnett [8], Fabian [9], Girshick [10], Gupta and Sobel [11], Mosteller [13] and Paulson [14] [18] Taylor and David [25], Truax [26]. They have discussed this problem from several different viewpoints such as slippage aspect grouping aspect and ranking aspect. Some of them appealed to sequential multiple decision procedures which were not necessary closed. On the other hand authors such as Armitage [1], Schneiderman [21], Schneiderman and Armitage [22], Sobel and Wald [23], Sobel [24] appealed to restricted or closed sequential procedures which, however, were not necessarily multiple decision procedure.

It was Paulson [19], [20] who presented a class of closed sequential multiple decision procedures for a set of significance level $\alpha$ in $0<\alpha<1$ and for a certain configuration of population means for which the probability by which the best population (having the largest means) among several normal populations is selected is larger than the prescribed value $1-\alpha$.

The object of this paper is to generalize the results of Paulson [19], [20] in two directions. In the first place we shall be concerned with a more general class of population distributions, that is, one parameter exponential distributions. In the second place we shall discuss with a configuration of population parameters which are more general than that which Paulson [19], [20] did substantially consider. Such a generalized configuration of population parameters is indeed both subtle and necessary 
in our generalized set-up dealing with one parameter exponential distribution.

It is shown in this paper that the essential aspect of our closed sequential statistical procedures (CSSP) can be established in the frame of additive family of sufficient statistics whose notion was introduced by Kitagawa [12]. It is also noted that the restrictions on population distributions are required to establish our results. However these restrictions are so mild that we can easily obtain various examples regarding normal distributions, $x^{2}$-distributions, Poisson distributions and binomial distributions.

\section{§. Definitions and introduction.}

Definitions. (I). Let $\Pi_{i}$ be a population whose probability density function with respect to a common measure $\mu$ over the real line is given by

$$
f\left(u_{i}, \tau_{i}\right) d \mu\left(u_{i}\right)=\exp \left\{\tau_{i} u_{i}+b\left(\tau_{i}\right)+a\left(u_{i}\right)\right\} d \mu\left(u_{i}\right),
$$

defined over $-\infty<u_{i}<\infty$ where $\tau_{i}$ is a real parameter whose value is unknown to us, where $b\left(\tau_{i}\right)$ and $a\left(u_{i}\right)$ are real valued known functions of $\tau_{i}$ and $u_{i}$, respectively, and where $b\left(\tau_{i}\right)$ has the first and the second derivatives $b^{\prime}\left(\tau_{i}\right)$ and $b^{\prime \prime}\left(\tau_{i}\right)$ which are continuous and $b^{\prime}\left(\tau_{i}\right)$ is strictly monotone decreasing with respect to $\tau_{i}$ in a certain finite interval of $\tau_{i}(i=1,2, \cdots, k)$. A population is said to be of an exponential type when its probability density function is the form (2. 1).

Notation. (I). Let order $k$ values $\tau_{1}, \tau_{2}, \cdots, \tau_{k}$ in the ascending order of magnitude, and write in the form $\tau_{(1)} \leqq \tau_{(2)} \leqq \cdots \leqq \tau_{(k)}$.

Definition. (II). In what follows in this paper, the best population among $k$ populations of exponential type means the population which has the largest parameter value $\tau_{(k)}$ in case $\tau_{(1)} \leqq \tau_{(2)} \leqq \cdots \leqq \tau_{(k)}$ and it means a populaton which has been selected by certain chance mechanism among $s$ populations whose values of the parameters are equal to $\tau_{(k)}$, when it holds true that $\tau_{(1)} \leqq \cdots \leqq \tau_{(k-s)}<\tau_{(k-s+1)}=\cdots=\tau_{(k)}$ for some $s>1$.

Notation. (II). We denote by $\delta_{\Delta}\left(\tau_{(k)} ; \lambda_{1}, \cdots, \lambda_{k-1}\right)=\delta_{\Delta}\left(\tau_{(k)}, \lambda\right)$ where $\lambda=\left(\lambda_{1}, \cdots, \lambda_{k-1}\right)$, and $\lambda_{i} \geqq 0, \quad(i=1,2, \cdots, k-1)$, a configuration of population parameters satisfying the conditions $\tau_{i}=\tau_{(k)}-\Delta-\lambda_{i}$ for a positive constant value $\Delta(i=1,2, \cdots, k-1)$.

In what follows we shall be concerned with a certain type of closed sequential statistical procedure whose specification will be given in $\S 3$. A general aspect of any closed sequential statistical procedure may be enunciated as follows:

(1) There is an assigned positive integer $N \geq 2$.

(2) There is an assigned sequence of positive integers $\left(m_{1}, m_{2}, \cdots\right.$, $\left.m_{N}\right)$.

(3) Let us consider $k$ populations and let us draw a sample of size $m_{1}$ from each population. Let us construct a rejection region from $k$ 
populations which can be determined by means of a preassigned constant of significance level $\alpha$ and observed values of the sample. Then we decide whether each population is rejected or not, by a test defined by the rejection region and by the sample.

(a) In this case, if only one population was not rejected, then we do not draw any more sample and we can and we shall decide that population as the best one.

(b) If more than one populations were left as the candidates for the best one, then we shall proceed to the following second stage.

(ii). In the second stage, let us consider those populations which were not rejected at the first stage enunciated in (i). Let us draw an additional sample of size $m_{2}$ from each population and make a pooled sample of size $m_{1}+m_{2}$ for each population. Let us construct a rejection region for populations which can be determined by means of a preassigned constant of significance level $\alpha$ and observed values of the sample. Then we decide whether each population is rejected or not, by a test defined by the rejection region and by the sample.

(a) In this case, if only one population was not rejected, then we do not draw any more sample and we can and we shall decide that population as to be the best one.

(b) If more than one populations were left as the candidates for the best one, then we shall proceed to the following third stage and so on.

$\left(l^{\circ}\right)$. $(3 \leqq l \leqq N)$. We continue these steps in a sequential way, so far as the best population has not been decided and the step number $l$ is smaller than a predetermined integer $N$.

$\left((N+1)^{\circ}\right)$. If more than one populations were left as the candidates for the best one at the $N$-th stage, we shall decide uniquely the best population among those populations which have not been rejected until the $N$-th stage, by use of certain chance mechanism.

\section{$\S 3$. The purpose of this paper.}

Definition. (III). We denote by $\widetilde{F}(T, d)$ a class of configuration $\delta_{\Delta}$ $\left(\tau_{(k)}, \lambda\right)$ of $k$ populations which have the probability density functions of exponential type satisfying the following conditions:

(1) The parameter $\tau_{(k)}$ belongs to a certain finite interval $T$.

(2) The set of $(k-1)$ parameter $\tau_{i}=\tau_{(k)}-\Delta-\lambda_{i}(i=1,2, \cdots, k-1)$ satisfies the condition that

$$
\operatorname{Min}_{1 \leqq i \geqq k-1}\left(b^{\prime}\left(\tau_{i}\right)-b^{\prime}\left(\tau_{(k)}\right)\right)=d\left(\tau_{(k)}, \lambda\right) \geqq d .
$$

Under these definitions and assumptions, the main results of this paper are concerned with the following two purposes (1) and (2):

(1) To give a family of closed sequential statistical procedure such that, for each of them, the probability by which the best population is selected 
is larger than $1-\alpha$ for each assigned $\alpha$ in $0<\alpha<1$ and for each set of $k$ populations of exponential type having a configurations $\mathfrak{F}(T, d)$, where the interval $T$ and the positive constant $d$ are arbitrarily preassigned. We shall denote such a class of closed sequential statistical procedures by CSSP $(\alpha ; \Delta, T, d)$. (2) To specify a closed sequential statistical procedure which belongs to a class $\operatorname{CSSP}(\alpha ; \Delta, T, d)$ and which has the minimum number of stages for all configurations belonging to $\mathfrak{F}(T, d)$. However it is hard to establish this object, therefore we shall present several examples in $\$ 7$. In what follows, the constants $k$ and $\Delta$ which are enunciated in the previous sections are assumed to be given and fixed.

\section{\$. The enunciation of the closed sequential statistical procedure.}

Now our closed sequential procedure, which is denoted by $S_{\theta}$ for each value of $\theta$ in the interval $0<\theta<e_{0}$, is defined as follows:

(0) Let us define

$$
N(\theta)=\frac{\log ((k-1) / \alpha)}{-\theta h_{0}(\theta) \Delta}
$$

and

$$
A^{(n)}(\theta)=K(\theta)+n \theta, n=1,2, \cdots, N(\theta),
$$

where we put

$$
K(\theta)=\frac{\log ((k-1) / \alpha)}{h_{0}(\theta) \Delta},
$$

and where $h_{0}(\theta)$ is given as a root of the equation (6.1.19) explained afterwards.

Since the size $m_{i}$ of sample drawn at the $i$-th stage is equal to a certain prescribed constant $m_{i}$ for $i=1,2, \cdots, N(\theta)$, we can and we shall assume that $m_{1}=m_{2}=\cdots=m_{N(\theta)}=1$ without loss of generality.

(i) In the first stage, let $\boldsymbol{u}_{i}^{(1)}=U_{i}^{(1)}$ be a statistic defined by a sample of size one drawn from the population $\Pi_{i}^{(1)},(i=1,2, \cdots, k)$ respectively. Let us arrange the set of $k$ values $U_{1}^{(1)}, \cdots, U_{k}^{(1)}$ in the ascending order of magnitude, and write them in the form $U_{(1)}^{(1)} \leqq \cdots \leqq U_{(k)}^{(1)}$. If the following relation holds true

$$
U_{(i)}^{(1)}<U_{(k)}^{(1)}+A^{(1)}(\theta),
$$

then we reject the population $\Pi_{(i)}^{(1)}$, in $1 \leq i \leqq k-1$. Now our statistical procedure proceeds in either one of two alternative ways: (i) (a) and (i) (b).

(i) (a) In this case, if only one population $\Pi_{(k)}^{(1)}$ was not rejected, then we do not draw any more sample and we can and we shall decide that population $\Pi_{(k)}^{(1)}$ as to be the best one.

(i) (b) If more than one populations were left as the candidates for 
the best one, then we shall proceed to the following second stage.

(ii) In the second stage, let us consider those populations which were not rejected at the first stage (i), and let $\left\{U_{j i}^{(1)}\right\}\left(i \cdots 1,2, \cdots, k_{1}\right)$ be a set of $k_{1}$ statistics each of which $U_{j i}^{(1)}$ came from the population $I_{j i}^{(1)}$ which was not rejected at the first stage (i), $\left(i=1,2, \cdots, k_{1}\right)$. Let us now rewrite $U_{j i}^{(1)}$ and $\Pi_{j i}^{(1)}$ as $W_{i}^{(2)}$ and $\Pi_{i}^{(2)}\left(i=1,2, \cdots, k_{1}\right)$, respectively. Let $u_{i}^{(2)}$ be a statistic defined by an additional sample of size one drawn from $\Pi_{i}^{(2)},\left(i=1,2, \cdots, k_{1}\right)$, and put that

$$
U_{i}^{(2)}=W_{i}^{(2)}+u_{i}^{(2)}, \quad\left(i=1,2, \cdots, k_{1}\right) .
$$

Let us arrange the set of $k_{1}$ values $U_{1}^{(2)}, \cdots, U_{k 1}^{(2)}$ in the ascending order of magnitude, and write them in the form $U_{(1)}^{(2)} \leq \cdots \leq U_{(k)}^{(2)}$. If the following relation holds true

$$
U_{(i)}^{(2)}<U_{(k)}^{(2)}+A^{(2)}(\theta),
$$

then we reject the population $I_{(i)}^{(2)}$ with the statistic $U_{(i)}^{(2)}\left(i=1,2, \cdots, k_{1}-1\right)$ respectively. Now our statistical procedure proceeds in either one of two alternative ways: (ii) (a) and (ii) (b).

(ii) (a) In this case, if only one population $\left.\Pi_{(k)}^{(2)}\right)$ was not rejected, then we do not draw any more sample and we can and we shall decide that population $\Pi_{(k 1)}^{(2)}$ as to be the best one.

(ii) (b) If more than one population were left as the candidates for the best one, then we shall proceed to the following third stage and so on.

$\left(l^{\circ}\right)(3 \leqq l \leqq N(\theta))$. In the $l$-th stage, let us consider those populations which were not rejected at the $(l-1)$-th stage and let $\left\{U_{j i}^{(l-1)}\right\}$ for $i=1,2$, $\cdots, k_{l-1}$ be a set of $k_{l-1}$ statistics each of which $U_{j i}^{(l-1)}$ came from the population $\Pi_{j i}^{(l-1)}$ which was not rejected at the $(l-1)$-th stage, $(i=1,2, \cdots$, $\left.k_{l-1}\right)$. Let us now rewrite $U_{j i}^{(l-1)}$ and $\Pi_{j \downarrow}^{(l-1)}$ as $W_{i}^{(l)}$ and $\Pi_{i}^{(l)}, i=1,2, \cdots k_{l}$ respectively. Let $u_{i}^{(l)}$ be a statistic defined by an additional sample of size one drawn from $\Pi_{i}^{(l)}\left(i=1,2, \cdots, k_{l-1}\right)$ and put that

$$
U_{i}^{(l)}=W_{i}^{(l)}+u_{i}^{(l)}, \quad\left(i=1,2, \cdots, k_{l-1}\right) .
$$

Let us arrange the set of $k_{l-1}$ values $U_{1}^{(1)}, \cdots, U_{k_{l-1}}^{(l)}$ in the ascending order of magnitude, aud write them in the form $U_{(1)}^{(l)} \leqq \cdots \leqq U_{\left(k_{l-1}\right)}^{(l)}$. If the following relation holds true

$$
U_{i}^{(l)}<U_{\left(k_{l-1}\right)}^{(l)}+A^{(l)}(\theta),
$$

then we reject the population $I_{i}^{(l)}$ with the statistic $U_{i}^{(l)}, \quad\left(i=1,2, \cdots, k_{l-1}\right)$ respectively. Our statistical procedure proceeds in either one of two alternative way: $\left(l^{\circ}\right)\left(\right.$ a) and $\left(l^{\circ}\right)($ b) .

$\left(l^{\circ}\right)$ (a) In this case, if only one population $\Pi_{\left(k_{l-1}\right)}^{(l)}$ was not rejected, then we do not draw any more sample and we can and we shall decide that population $\Pi_{\left(k_{l-1}\right)}^{(l)}$ as to be the best one.

$\left(l^{\circ}\right)(b)$ If more than one populations were left as the candidates for the best one, then we shall proceed to the following stage. We continue 
these steps in a sequential ways, so far as the best population has not been decided and until the step number $l$ is smaller than or equal to a predetermined integer $N(\theta)$. If we came to the case of $\left(N(\theta)^{\circ}\right)$ (b), then we proceed to the following $(N(\theta)+1)$-th stage.

$\left((N(\theta)+1)^{\circ}\right)$. In this stage, let us consider those populations which were not rejected at the $N(\theta)$-th stage and let $\left\{U_{j i}^{(N(\theta))}\right\}$ for $i=1,2, \cdots, k_{N(\theta)}$ be a set of $k_{N^{\prime}(\theta)}$ statistics each of which $U_{j i}^{(N(\theta))}$ came from the populations $\Pi_{j i}^{(N(\theta))}$ which was not rejected at the $N(\theta)$-th stage, $\left(i=1,2, \cdots, k_{N(\theta)}\right)$. Let us now rewrite $U_{j i}^{\langle N(\theta)\rangle}$ and $\Pi_{j i}^{(N(\theta))}$ as $W_{i}^{(N(\theta)+1)}$ and $\Pi_{i}^{(N(\theta)+1)} i=1,2, \cdots, k_{N(\theta)}$ respectively. Let $U_{i}^{(N(\theta)+1)}$ be a statistic defined by an additional sample of size one drawn from $\Pi_{i}^{(N(\theta)+1)}\left(i=1,2, \cdots, k_{(N(\theta)+1)}\right)$ and put that

$$
U_{i}^{(N(\theta)+1)}=W_{i}^{(N(\theta)+1)}+\boldsymbol{u}_{i}^{(N(\theta)+1)},
$$

$i=1,2, \cdots, k_{N(\theta)}$. Let us arrange the set of $k_{N(\theta)}$ values $U_{1}^{(N(\theta)+1)}, \cdots, U_{k_{N(\theta)}^{(N)(\theta)+1)}}$ in the ascending order of magnitude, and write them in the form $U_{(1)}^{(N())+1)} \leqq \cdots$

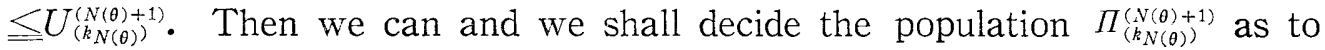
be the best one.

In this case, when we use the closed sequential procedure $S_{\theta}$ for $k$ populations each of which belongs to the exponential type, we can obtain main theorem which we shall enunciate it in the next section.

\section{$\S 5$, The enunciation of theorem.}

In order to enunciate a main theorem, let us introduce the following notations :

Let us denote by $P\left[\Pi_{(k)} S_{\theta}, \delta_{\Delta}\left(\tau_{(k)}, \lambda\right)\right]$ the probability by which the best populations of exponential type is rejected by a certain specified closed sequential statistical procedure $S_{\theta}$ under the configuration of population parameters $\delta_{\Delta}\left(\tau_{(k)}, \lambda\right)$.

Then we have the following

Theorem. For a set of constants $\{k, \alpha, \theta, \Delta\}$ which were assigned previously in $0<\alpha<1, k \geq 2,0<\theta<d$ and $\Delta>0$, we have

$$
P\left[\Pi_{(k)} S_{\theta}, \delta_{\Delta}\left(\tau_{(k)}, \lambda\right)\right] \leqq \alpha .
$$

\section{\$. Proof of the theorem.}

\section{1. Preliminaries.}

In this section let us prove the main theorem in case when $k=2$ and when $k>2$ separetely. This is done not only because the case when $k=2$ is important in itself, but also because the probability by which the best population among $k$ populations of exponential type having a configuration of $k$ population parameters $\delta_{4}\left(\tau_{(k)}, \lambda\right)$ is rejected is not larger than the $(k-1)$ times of the probability by which the best population between two populations is rejected under the same parameter configuration, as we shall 
varify later.

Before we enter into the proof of the theorem in the case when $k=2$, let us enunciate the following preparations:

First of all let us assume $\tau_{2}>\tau_{1}$ and let us put

$$
f\left(u_{i, j}, \tau_{h}\right)=\exp \left\{\tau_{i} u_{i, j}-b\left(\tau_{h}\right)+a\left(u_{i, j}\right) !,\right.
$$

for $i, h=1,2 ; j=1,2, \cdots$ and put that for each $\theta$ in $0<\theta<d$

$$
z_{j}=\log \left\{\begin{array}{l}
f\left(u_{2, j}, \tau_{2}\right) f\left(u_{1, j} \div \theta, \tau_{1}\right) \\
f\left(u_{2, j}, \tau_{1}\right) f\left(u_{1 j}+\theta, \tau_{2}\right)
\end{array}\right\}, j=1,2, \cdots
$$

Lemma 1. (Wald $[27]$, p. 158) Let $z$ be a random variable such that the following three conditions are fulfilled:

(A) The expected value $E\{z\}$ exists and is not equal to 0 .

(B) There exists a positive $\varepsilon$ such that $P[\exp z<1-\varepsilon]>0$ and $P$ $[\exp z>1+\varepsilon]>0$.

(C) For any real value $h$ the expected value $E\{\exp (h z)\}=g(h)$ exists.

Then there exists one and only one real value $h_{0} \neq 0$ such that

$$
E\left\{\exp \left(h_{0} z\right)\right\}=1 \text {. }
$$

Lemma 2. Let $\left\{z_{j}\right\}$ be a set of independent random variables for which (6.1.1) and (6.1.2) are defined. Then each random variable $z_{j}$ in a set of $\left\{z_{j}\right\}$ satisfies the three conditions (A), (B) and (C) enunciated in Lemma 1 .

Proof: $\operatorname{Ad}(\mathrm{A})$. For each $z_{j}$ we have

$$
\begin{aligned}
E\left\{z_{j}\right\} & =\left(\tau_{2}-\tau_{1}\right) E\left\{u_{2, j}-u_{1, j}-\theta\right\} \\
& =\left(\tau_{2}-\tau_{1}\right)\left\{b^{\prime}\left(\tau_{1}\right)-b^{\prime}\left(\tau_{2}\right)-\theta\right\} \\
& \geq \Delta(d-\theta)>0,
\end{aligned}
$$

under the configuration $\delta_{\Delta}\left(\tau_{2}, \lambda_{1}\right)$, where $\Delta$ and $d$ are two specified positive constant values given in $\$ 3$.

$\mathrm{Ad}(\mathrm{B})$. For any assigned constant value of $\theta$ in $0<\theta<d$ and for any assigned constant value of $\varepsilon$ in $0<\varepsilon<1$, if we have that the following probability

(6. 1. 5)

$$
\begin{aligned}
& P\left[\exp z_{j}<1-\varepsilon \delta_{\Delta}\left(\tau_{2}, \lambda_{1}\right)\right] \\
= & P\left[u_{2, j}<u_{1, j}+\theta+\left(\tau_{2}-\tau_{1}\right)^{-1} \log (1-\varepsilon) \delta_{\Delta}\left(\tau_{2}, \lambda_{1}\right)\right]
\end{aligned}
$$

is equal to 0 , then the following equation holds true

(6. 1.6)

$$
P\left[u_{2, j} \geq u_{1, j}+\theta+\left(\tau_{2}-\tau_{1}\right)^{-1} \log (1-\varepsilon) \delta_{\Delta}\left(\tau_{2}, \lambda_{1}\right)\right]=1,
$$

for any constant values $\theta$ and $\varepsilon$ in $0<\theta<d$ and in $0<\varepsilon<1$. This contradicts to the definition of the measure $\mu$, since the random variables $u_{1, j}$ and $u_{2, j}$ are mutually independent.

Similarly we can verify the second part of (B).

$\operatorname{Ad}(\mathrm{C})$. For any real value of $h$ we have 
(6. 1. 7) $g(h)=E\left\{\exp \left\{h\left(\tau_{2}-\tau_{1}\right)\left(u_{2, j}-u_{1, j}-\theta\right)\right\}\right\}$

$$
\begin{aligned}
= & \int_{-\infty}^{\infty} \exp :\left(\tau_{2}-\tau_{1}\right) h+\tau_{2}\left\{u_{2, j}+b\left(\tau_{2}\right)+a\left(u_{2, j}\right)\right\} d \mu\left(u_{2, j}\right) \\
& \times \int_{-\infty}^{\infty} \exp \left\{\left\{\tau_{1}-\tau_{2}\right) h+\tau_{1} ; u_{1, j}+b\left(\tau_{1}\right)+a\left(u_{1, j}\right)\right\} d \mu\left(u_{1, j}\right) \\
& \times \exp \left\{\left(\tau_{1}-\tau_{2}\right) h \theta\right\} \\
= & \exp \left\{b\left(\tau_{1}\right)+b\left(\tau_{2}\right)+\left(\tau_{1}-\tau_{2}\right) h \theta-b\left(\left(\tau_{2}-\tau_{1}\right) h+\tau_{2}\right)\right. \\
& \left.-b\left(\left(\tau_{1}-\tau_{2}\right) h+\tau_{1}\right)\right\} .
\end{aligned}
$$

The immediate consequence of Lemma 2 is the following

Lemma 3. Let $\left\{z_{j}\right\}$ be a set of independent random variables for which (6. 1. 1) and (6.1.2) are defined. Then there exists one and only one real value $h_{0} \neq 0$ such that

$$
E\left\{\exp \left\{h_{0} z_{j}\right\}\right\}=-1,(j=1,2, \cdots) \text {. }
$$

Lemma 4. The value $h_{0}$ in (6.1.8) is negative.

Proof: In virtue of (6.1.4), the proof is immediate from the proof of Lemma 1 (Wald [27], p. 159).

The immediate consequence of Lemma 3 and Lemma 4 is the following

Lemma 5. Let $B(\theta)$ be any assigned positive function defined over the interval $0<\theta<d$. Let us terminate the sampling at the $n$-th stage, if (6. 1. 9)

$$
B(\theta)<\exp \left\{Z^{(n)}\right\}, \quad(m=1,2, \cdots, n-1),
$$

and

(6. 1. 10)

$$
\exp \left\{Z^{(n)}\right\} \subseteq B(\theta)
$$

where $z_{j}, j=1,2, \cdots, n$ are defined by (6. 1. 1) and (6. 1.2), and where we have put $Z^{(n)}=\sum_{j=1}^{n} z_{j}$.

Then we have

(i) For each $\theta$ in $0<\theta<d$ the value of $h_{0}(\theta)$ is determined so that $h_{0}(\theta)<0$ and that

$$
E\left\{\exp \left\{Z^{(n)} h_{0}(\theta)\right\}\right\}=1 .
$$

(2) For every $n \geq 1$ we have

$$
B(\theta)^{h_{0}(\theta)} \int \cdots \cdots \int_{i=1}^{2} \prod_{j=1}^{n} f\left(u_{i, j}, \tau_{j}\right) d \mu\left(u_{i, j}\right) \leqq 1 .
$$

Proof : Ad (i) Since $\left\{z_{j}\right\}$ is a set of independent and identically distributed random variables, we have

$$
E\left\{\exp \left\{Z^{(n)} h_{0}(\theta)\right\}\right\}=\prod_{j=1}^{n} E\left\{\exp \left\{z_{j} h_{0}(\theta)\right\}\right\}=1 \text {. }
$$

$\operatorname{Ad}(2)$. In virtue of (1), we have 
(6. 1. 14)

$$
\begin{aligned}
1 & =E\left\{\exp \left\{Z^{(n)} h_{0}(\theta)\right\}\right\} \\
& =\int \cdots \cdots \int \exp \left\{Z^{(n)} h_{0}(\theta)\right\} \prod_{i=1}^{2} \prod_{j=1}^{n} f\left(u_{i, j}, \tau_{j}\right) d \mu\left(u_{i, j}\right) \\
& \exp \left\{\boldsymbol{Z}^{(n)}\right\} \leqq B(\theta) \\
& \geq B(\theta)^{h_{0}(\theta)} \int \cdots \cdots \prod_{i=1}^{2} \prod_{j=1}^{n} f\left(u_{i, j}, \tau_{j}\right) d \mu\left(u_{i, j}\right) . \\
& \quad \exp \left\{\boldsymbol{Z}^{(n)}\right\} \leqq B(\theta)
\end{aligned}
$$

Lemma 6. (Paulson [19], p.176) If $\left\{Y_{j}\right\}$ is a sequence of independent and identically distributed random variables with a negative expectation and $b>0$, then we have

$$
P\left[\sum_{j=1}^{n} Y_{j}>b \text { for some } n<\infty\right] \leqq \exp \left\{-h_{0} b\right\},
$$

where $h_{0}$ is the non-zero root of $E\{\exp \{h Y\}\}=1$.

Lemma 7. Let us put $Z^{(n)}=\sum_{j=1}^{n} z_{j}$ for $n=1,2, \cdots$, where $\left\{z_{j}\right\}$ are defined by (6. 1. 1) and (6. 1.2). Let $\stackrel{j=1}{B}(\theta)$ be any assigned positive, however smaller than one, function defined over the interval $0<\theta<d$. Let $h_{0}(\theta)$ be a value such that $h_{0}(\theta)<0$ and that (6.1.11) is satisfied. Then we have (6. 1. 16) $P\left[Z^{(n)} \leqq \log B(\theta)\right.$ for some $\left.n<\infty\right] \leqq B(\theta)^{-h_{0}(\theta)}$

Proof : In Lemma 6, let us put that $b=-\log B(\theta)$ and $Y_{j}=-z_{j}(j=1$, $2, \cdots)$. By use of $(6.1 .4)$, the relation (6.1.16) follows immediately from the relation $(6.1 .15)$.

Lemma 8. For each assigned $\theta$ in $0<\theta<d$ and for any assigned $\alpha$ in $0<\alpha<1$, let us assume that the following relation

$$
-\log B(\theta) \geqq h_{0}(\theta)^{-1} \log \alpha
$$

hold true. Then we have

(6. 1. 18)

$$
P\left[Z^{(n)} \leqq \log B(\theta) \text { for some } n<\infty\right] \leqq \alpha .
$$

Proof: The proof of this lemma follows from Lemma 7 immediately.

Lemma 9. For each assigned $\theta$ in $0<\theta<d$, the root $h_{0}(\theta)$ defined by (6. 1. 11) is given by the root of the following functional equation

$$
b\left(\tau_{1}\right)+b\left(\tau_{2}\right)=b\left(\tau_{1}^{*}\right)+b\left(\tau_{2}^{*}\right)+\theta h_{0}(\theta)\left(\tau_{2}-\tau_{1}\right),
$$

where

(6. 1.20$)$

$$
\begin{aligned}
& \tau_{1}^{*}=\tau_{1}+\left(\tau_{1}-\tau_{2}\right) h_{0}(\theta), \\
& \tau_{2}^{*}=\tau_{2}+\left(\tau_{2}-\tau_{1}\right) h_{0}(\theta) .
\end{aligned}
$$

(6. 1. 21)

Proof : For each assigned $\theta$ in $0<\theta<d$, the proof follows immediately 
from the substitution of $h_{0}(\theta)$ to $h$ in (6. 1. 7).

6. 2. The proof of the theorem in the case when $k=2$.

First of all, in order to prove the theorem when $k=2$ let us introduce the following notations for the sake of convenience:

For each $l(1 \leqq l \leqq N(\theta)+1)$, let us denote by $D_{l}^{(l)}((1,2) ; 1)$ the stochastic event by which both population $I_{1}$ and $I_{2}$ are not rejected before the $l$-th stage and which the population $\Pi_{2}$ is rejected at the $l$-th stage by our statistical procedure $S_{\theta}$. Let us denote by $D_{N(\theta)+1}((1,2) ; 1)$ the stochastic event by which the population $I_{2}$ is rejected at a certain stage by our statistical procedure $S_{\theta}$.

In this connection let us denote by $P\left[\Pi_{(2)} S_{\theta}, \delta_{4}\left(\tau_{(2)}, \lambda\right)\right]$ the probability by which the population $\Pi_{2}$ is rejected by our statistical procedure $S_{\theta}$, under the configuration of population parameters $\delta_{\Delta}\left(\tau_{(2)}, \lambda\right)$. Then we have the following

Theorem. We have for each assigned $\alpha$ in $0<\alpha<1$

$$
P\left[\Pi_{(2)}: S_{\theta}, \delta_{\Delta}\left(\tau_{(2)}, \lambda\right)\right] \leqq \alpha .
$$

Proof : Firstly let us consider the case when $\tau_{2}=\tau_{(2)}$. Then we have

$$
\begin{aligned}
& P\left[\Pi_{(2)} S_{\theta}, \delta_{\Delta}\left(\tau_{(2)}, \lambda\right)\right] \\
= & P\left[D_{N(\theta)+1}((1,2) ; 1) S_{\theta}, \delta_{\Delta}\left(\tau_{(2)}, \lambda\right)\right] \\
= & P\left[D_{n}^{(n)}((1,2) ; 1) \text { for some } n(1 \leqq n \leqq N(\theta)+1) \mid S_{\theta}, \delta_{\Delta}\left(\tau_{(2)}, \lambda\right)\right] \\
= & P\left[\sum_{i=1}^{n} z_{i}<(\Delta+\lambda) K(\theta) \text { for some } n<\infty \mid S_{\theta}, \delta_{\Delta}\left(\tau_{(2)}, \lambda\right)\right],
\end{aligned}
$$

where we have put

$$
z_{i}=\left(\tau_{2}-\tau_{1}\right)\left(u_{2, i}-u_{1, i}-\theta\right), \quad(i=1,2, \cdots) .
$$

For each assigned $\theta$ in $0<\theta<d$, let us consider the root $h_{0}(\theta)$ of the following equation
(6. 2. 4)
$E\left[\exp \left(z h_{0}(\theta)\right)\right]=1$.

Since we have

$$
\begin{aligned}
E[z] & =\left(\tau_{2}-\tau_{1}\right)\left(b^{\prime}\left(\tau_{1}\right)-b^{\prime}\left(\tau_{2}\right)-\theta\right) \\
& \geq \Delta(d-\theta)>0,
\end{aligned}
$$

there exists one and only one negative root $h_{0}(\theta)$ of (6.2.4) in virtue of Lemma 4 . In virtue of $(6.1 .18)$ the probability in the right hand side of (6. 2. 2) does not exceed the following

$$
\begin{aligned}
& \exp \left\{-h_{0}(\theta)(\Delta+\lambda) K(\theta)\right\} \\
= & \exp \{-(\Delta+\lambda) / \Delta \log (1 / \alpha)\} \leqq \alpha,
\end{aligned}
$$

where $h_{0}(\theta)$ is calculated from (6. 1. 19) (6.1.21). 
In case when $\tau_{1}=\tau_{(2)}$, we can also verify the theorem by the similar discussions to that in case $\tau_{2}=\tau_{(2)}$.

6. 3. The proof of the theorem in case when $k>2$.

Let us denote by $\delta_{\Delta}\left(\tau_{k}, \lambda\right)$ the parameter configuration $\tau_{(k)}=\tau_{k}=\tau_{j}+\Delta$ $+\lambda_{j}, \lambda_{j} \geq 0$ for $\mathrm{j}=1,2, \cdots, k-1$. Paulson [19] has used the following result under our notations.

Lemma 10. (Paulson [19], p. 176) we have

(6. 3. 1) $P\left[\Pi_{k} S_{\theta}, \delta_{\Delta}\left(\tau_{k}, \lambda\right)\right]$

$$
\begin{aligned}
& \leq \sum_{j=1}^{k-1} P\left[U_{k}^{(n)}-U_{j}^{(n)}<A^{(n)}(\theta) \text { for some } n(1 \leqq n \leqq N(\theta)+1) S_{\theta},\right. \\
& \left.\quad \delta_{\Delta}\left(\tau_{k}, \lambda\right)\right] .
\end{aligned}
$$

Lemma 11. We have

$$
P\left[I_{(k)} \mid S_{\theta}, \delta_{\Delta}\left(\tau_{(k)}, \lambda\right)\right]=P\left[I_{k} \mid S_{\theta}, \delta_{\Delta}\left(\tau_{k}, \lambda\right)\right] .
$$

Proof: Since our closed sequential statistical procedure $S_{\theta}$ defined by $\S 4$ is symmetric with regard to $\tau_{(k)}$, the proof follows immediately.

Hence we have the following main theorem enunciated in $\$ 5$.

Theorem. For a set of constants $\{k, \alpha, \theta, \Delta\}$ which were assigned previously in $0<\alpha<1, k \geqq 2,0<\theta<d$ and $\Delta>0$, we have

$$
P\left[\Pi_{(k)}, S_{\theta}, \delta_{\Delta}\left(\tau_{(k)}, \lambda\right)\right] \leqq \alpha .
$$

Proof: The proof is immediate from Lemma 10, Lemma 11 and (6. 2. 1).

Lemma 12. For any assigned integers $j_{i}$ in $1 \leqq j_{i} \leqq k-1, i=1,2 ; j_{1} \neq j_{2}$, we have

$$
\text { (6. 3. 4) } \begin{aligned}
& P\left[U_{k}^{(n)}-U_{j_{1}}^{(n)}<A^{(n)}(\theta) \text { for some } n(1 \leqq n \leqq N(\theta)+1) S_{\theta}, \delta_{\Delta}\left(\tau_{k}, \mathbf{0}\right)\right] \\
= & P\left[U_{k}^{(n)}-U_{j 2}^{(n)}<A^{(n)}(\theta) \text { for some } n(1 \leqq n \leqq N(\theta)+1) S_{\theta}, \delta_{\Delta}\left(\tau_{k}, \mathbf{0}\right)\right]
\end{aligned}
$$

Proof : For any assigned numbers $n, j_{1}$ and $j_{2}$ in $1 \leqq n \leqq N(\theta)+1$ and in $1 \leqq j_{i} \leqq k-1, i=1,2 ; j_{1} \neq j_{2}$, the random variables $U_{j 1}^{(n)}$ and $U_{j 2}^{(n)}$ are identically distributed under the parameter configuration $\delta_{\Delta}\left(\tau_{k}, \mathbf{0}\right)$. Hence the proof is immediate.

Corollary. We have

$$
\begin{aligned}
& P\left[\Pi_{(k)} \quad S_{\theta}, \delta_{\Delta}\left(\tau_{(k)}, \mathbf{0}\right)\right] \\
\leqq & (k-1) P\left[\Pi_{(2)} S_{\theta}, \delta_{\Delta}\left(\tau_{(2)}, \mathbf{0}\right)\right] .
\end{aligned}
$$

Proof: The proof is immediate from Lamma 11 and Lemma 12.

\section{§. Examples.}

Let us give several examples concerning with the specification of the closed sequential statistical procedure which has the minimum number of stages with regard to $\theta$ in $0<\theta<d$, where $d$ is a preassigned constant 
which was defined by $\S 3$.

Example 1. (Mean of normal distribution). Let $\left\{u_{i, j}\right\},(i=1,2, \cdots, k$; $j=1,2, \cdots)$ be $k$ sequences of independent statistics having their probability density function of (2.1), provided that

$$
\tau_{i}=\mu_{i}, \quad b\left(\tau_{i}\right)=-\tau_{i}^{2} / 2, \quad(i=1,2, \cdots, k) .
$$

In this case let us determine a value of $N(\theta)$, defined by (4. 1), with regard to $\theta$ in $0<\theta<A$, where $A$ is a preassigned positive constant defined by $\$ 2$.

In virtue of (6. 1. 19) we have

$$
h_{0}(\theta)=-1+\theta / \Delta,
$$

and we have

$$
\operatorname{Min}_{0<\theta<\Delta}\left\{\theta h_{0}(\theta)\right\}=\Delta / 4
$$

therefore we have

$$
\operatorname{Min}_{0<\theta<\Delta} N(\theta)=4 \log ((k-1) / \alpha) / \Delta^{2} .
$$

Example 2. (Variance of normal distribution). Let $\left\{u_{i, j}\right\},(i=1,2, \cdots, k$; $j=1,2, \cdots)$ be $k$ sequences of independent statistics having their probability density function (2.1), provided that

$$
\tau_{i}=-\left(2 \sigma_{i}^{2}\right)^{-1},\left(\sigma_{i}>0\right), b\left(\tau_{i}\right)=2 \log \left(-\tau_{i}\right),
$$

$(i=1,2, \cdots, k)$. In virtue of $(8.1 .19)$ we have

(7. 6) $-\theta h_{0}(\theta)=\log \left\{\left(\tau_{(k)}+\Delta h_{0}(\theta)\right)\left(\tau_{(k)}-\Delta h_{0}(\theta)\right) /\left(\left(\tau_{(k)}-\Delta\right) \tau_{(k)}\right)\right\} /(2 \Delta)$.

Now let us put that

$$
\theta_{0,2}=\Delta^{-1} \log \left\{\left(2 \tau_{(k)}-\Delta\right)^{2} /\left(4 \tau_{(k)}\left(\tau_{(k)}-\Delta\right)\right)\right\},
$$

Then we have

$$
d_{2}=\left(1 /\left(\tau_{(k)}-\Delta\right)-1 / \tau_{(k)}\right) / 2 \text {. }
$$

$$
\operatorname{Min}_{0<\theta<d_{2}} N(\theta)=2 \log ((k-1) / \alpha) /\left(\theta_{0,2} \Delta\right) .
$$

Example 3. (Scale parameter of exponential distribution). Let $\left\{\boldsymbol{u}_{i, j}\right\}$, $(i=1,2, \cdots, k ; j=1,2 \cdots)$ be $k$ sequences of independent statistics having their probability density function of $(2.1)$, provided that

$$
\tau_{i}=-\lambda_{i}^{-1}, \quad\left(\lambda_{i}>0\right), b\left(\tau_{i}\right)=\log \left(-\tau_{i}\right), \quad(i=1,2, \cdots, k) .
$$

By means of the similar method used in Example 2, we have the following minimum value of $N(\theta)$ with regard to $\theta$ in $0<\theta<d_{3}$, where we put

$$
d_{3}=1 /\left(\tau_{(k)}-\Delta\right)-1 / \tau_{(k)} \text {. }
$$


(7. 12)

$$
\operatorname{Min}_{0<\theta<d_{3}} N(\theta)=2 \log ((k-1) / \alpha) /\left(\theta_{0,3} \mathcal{L}\right) .
$$

where we have put

$$
\theta_{0,3}=\frac{2}{\Delta} \log \left\{\begin{array}{c}
\left(2 \tau_{(k)}-\Delta\right)^{2} \\
4 \tau_{(k)}\left(\tau_{(k)}-\Delta\right)
\end{array}\right\} .
$$

Example 4. (Poisson distribution). Let $\left\{u_{i, j}\right\}, i=1,2, \cdots, k ; j=1,2, \cdots$ ) be $k$ sequences of independent statistics having their probability density function of (2.1), provided that

$$
\tau_{i}=\log \lambda_{i}, \quad\left(\lambda_{i}>0\right), b\left(\tau_{i}\right)=-\lambda_{i}, \quad(i=1,2, \cdots, k) .
$$

Let us put that

$$
\begin{aligned}
& \theta_{0,4}=2\left[\exp \left(\tau_{(k)} / 2\right)-\exp \left\{\left(\tau_{(k)}-\Delta\right) / 2\right\}^{2}\right] / \Delta, \\
& d_{4}=(1-\exp \{-\Delta\}) \exp \left\{\tau_{(k)}\right\} .
\end{aligned}
$$

By means of the similar method used in the previous examples, we have the following

$$
\operatorname{Min}_{0<\theta<d_{4}} N(\theta)=2 \log ((k-1) / \alpha) /\left(\theta_{0,4} \Delta\right) .
$$

Example 5. (Binomial distribution). Let $\left\{u_{i, j}\right\},(i=1,2, \cdots, k ; j=1,2, \cdots)$ be $k$ sequences of independent statistics having their probability density function of (2. 1), provided that

(7. 18) $\tau_{i}=\log \left(p_{i} /\left(1-p_{i}\right)\right),\left(0<p_{i}<1\right), b\left(\tau_{i}\right)=\log \left(1-p_{i}\right),(i=1,2, \cdots, k)$.

By means of the similar method used in the previous examples, we have the following. Let us put that

$$
\theta_{0,5}=\frac{2}{\Delta} \log \left\{\frac{\left(1+\exp \left\{\tau_{(k)}\right\}\right)\left(1+\exp \left\{\tau_{(k)}-\Delta\right\}\right)}{\left(1+\exp \left(\tau_{(k)}-\Delta / 2\right)\right)^{2}}\right\}
$$

(7. 20) $d_{5}=\left(1+\exp \left\{\tau_{(k)}\right\}\right)^{-2} \exp \left\{\tau_{(k)}\right\}-\left(1+\exp \left\{\tau_{(k)}-\Delta\{)^{-2} \exp \left\{\tau_{(k)}-\Delta\right\}\right.\right.$, then we have

$$
\operatorname{Min}_{0<\theta<d_{j}} N(\theta)=2 \log ((k-1) / \alpha) /\left(\theta_{0,5} \Delta\right) .
$$

\section{Acknowledgement.}

The author would like to express his hearty thanks to Professor $T$. Kitagawa of Mathematical institute, Kyushu University for his encouragements and criticisms during this paper was prepared.

\section{Ref erences.}

[1] Armitage, P.: Restricted sequenticl procedures, Biometrika, 44 (1957), 9-26.

[2] Bahadur, E.: On c problem in the theory of $k$ populctions, Ann. Math. Stat., 21 (1950), 362- 
375.

3] Bahadur, E. and Robbins, H.: The problem of the grecter mesn, Ann. Math. Stat., 21 (1950), 469-487.

[4] Bechhofer, R. E.: A single sample multiple decision procedur for ranking means of normal populctions with known varicnce, Ann. Math. Stat., 25 (1954), 16-39.

[5] Bechhofer, R. E.: A sequenticl multiple-decision procedure for selecting the best one of several normal populctions with a common unknown varicnce, and its use with vorious experimentcl designs, Biometrics, 14 (1958), 408-429.

[6] Bechhofer, R. E. and Blumenthal, S.: A sequential multiple-decision procedure for selecting the best one of several normal populations with a common unknown varicnce, II; Monte Carlo sampling results and new computing formulc, Biometrika, 18 (1962), 52-67.

[7] Bechhofer, R. E., Dunnett, C. W. and Sobel, M.: A two-sample multiple decision procedure for ranking means of normal populctions with a common unknown variances with $\mathrm{c}$ common unknown vcricnces, Biometrika, 41 (1954), 170-176.

[8] Dunnett, C. W.: A multiple comparison procedure for comparing severcl treatments with $\mathrm{C}$ control, Jour. Amer. Stat. Assn., 50 (1955), 1096-1121.

[9] Fabian, V.: On multiple decision methods for renking population mecns, Ann. Math. Stat., 33 (1962), 248-254.

[10] Girshick, M. A.: Contributions to the theory of sequenticl analysis, Ann. Math. Stat., 17 (1946), 123-143.

[11] Gupta, S. S. and Sobel, M.: On selecting a subset contcining the populction with the smallest verience, Biometrika, 49 (1962), 495-507.

[12] Kitagawa, T.: Successive process of statistical inference cssocicted with cn additive fomily of sufficient stotistics, Bull. Math. Stat., 7 (1957), 92-112.

[13] Mosteller, F.: A $k$-sample slipprsge test for an extreme population, Ann. Math. Stat., 19 (1948), $58-65$.

[14] Paulson, E.: A Multiple decision procedure for certain problem in the anslysis of variance, Ann. Math. Stat., 20 (1949), 95-98.

[15] Paulson, E.: On the comparison of several experimental categories with a control, Ann. Math. Stat., 23 (1952), 239-246.

[16] Paulson, E.: An optimum solution to the $k$ ssmple slippsge problem for the normal distribution, Ann. Math. Stat., 23 (1952), 610-616.

[17] Paulson, E. : A sequenticl procedure for compairing several experimental categories with $a$ stcndord or control, Ann. Math. Stat., 33 (1962), 438-443.

[18] Paulson, E.: A sequential procedure for choosing one of $k$ hypotheses concerning the unknown mean of c normal distribution, Ann. Math. Stat., 34 (1963), 549-554.

[19] Paulson, E.: A sequential procedure for selecting the population with the lorgest mecns from $k$ normal population Ann. Math. Stat., 35 (1964), 174-180.

[20] Paulson, E.: Sequential estimation and closed sequential decision procedures, Ann. Math. Stat., 35 (1964), 1048-1058.

[21] Schneiderman, M. A.: Closed sequenticl t-test, Biometrika, 49 (1962), 359-366.

[22] Schneiderman, M. A. and Armitage, P.: A family of closed sequeutiol procedures, Biometrika, 49 (1962), 41-56.

[23] Sobel, M. and Wald, A.: A sequenticl decision procedure for choosing one of three hypotheses concerning with unknown mesn of e normal distribution, Ann. Math. Stat., 20 (1949), 502-522.

[24] Sobel, M.: Sequential procedures for selecting the best exponential population, Proceedings of the Third Berkeley Symposium on Mathematical Statistics and Probability, Univ. of California Press, Berkeley, Vol. 5 (1956) 99-110.

[25] Taylor, R. J. and David, H. A.: A multi-stcge procedure for the selection of the best of several populctions, Jour. Amer. Stat. Assn., 57 (1962), 785-795.

[26] Truax, D. R.: An optimum slippage test of the varicnces of $k$ normal populctions, Ann. Math. Stat., 28 (1953), 669-674.

[27] Wald, A.: Sequential analysis, Wiley, New York (1947). 